\title{
Die empirische Erfassung von Medien-Frames
}

\author{
Jörg Matthes / Matthias Kohring
}

Die Studie greift die Probleme bei der empirischen Erfassung von Medien-Frames auf. Zunächst werden die bisherigen Methoden dargestellt, an denen vor allem die mangelnde Reliabilität und Validität zu kritisieren ist. Anschließend wird ein Verfabren zur Erfassung von Medien-Frames vorgestellt und beispielhaft an der von Entman (1993) vorgelegten Definition umgesetzt. Diese Methode gebt davon aus, dass sich die durch eine Inhaltsanalyse erfassten und vorab definierten Frame-Elemente in einer charakteristischen Weise gruppieren und somit clusteranalytisch zu einem Frame zusammenfassen lassen. Das Verfabren wird an einem internationalen Datensatz zur Biotechnologieberichterstattung demonstriert. Ergebnis sind fünf differenzierbare Frames (Landwirtschaftlicher Nutzen, Medizinischer Nutzen, Forschung in der Biomedizin, Landwirtschaft: Pro E Contra, Ethische Abwägung) sowie ein Frame (Diffuser allgemeiner Fortschritt), der sich schwieriger beschreiben lässt. Die vorgeschlagene Methode wird im Hinblick auf ihre Implikationen für die zukünftige Forschung diskutiert.

Keywords: Medien-Frames, Methoden, Biotechnologie, Inhaltsanalyse, internationaler Medienvergleich

\section{Einleitung}

Die Frame-Forschung hat in den letzten Jahren einen regelrechten Boom erlebt, der in verschiedenen Forschungsdisziplinen wie Soziologie, Politikwissenschaft, Psychologie und nicht zuletzt in der Kommunikations- und Medienwissenschaft seinen Niederschlag fand (vgl. Entman 1993; Iyengar 1991; McCombs/Llamas/Lopez-Escobar/Rey 1997; Pan/Kosicki 1993; Reese/Gandy/Grant 2001; Scheufele 1999). Die Zunahme an Forschungsarbeiten ist zunächst ein Zeugnis für die Relevanz und Aktualität des Ansatzes. Zugleich zeigt sich aber auch, dass derzeit weder ein theoretischer Konsens in der Frame-Forschung existiert, noch überhaupt der Stellenwert des Ansatzes innerhalb der Kommunikations- und Medienwissenschaft geklärt ist. Der Framing-Ansatz wird deshalb auch als zerstreutes Forschungsgebiet bezeichnet (vgl. Entman 1993: 51; Scheufele 1999: 118). Frames werden an vier verschiedenen Punkten des Kommunikationsprozesses lokalisiert: beim Kommunikator (1), beim Journalisten (2), beim Medieninhalt/Text (3) und beim Rezipienten (4).

(1) Kommunikatoren wie z. B. politische Akteure oder soziale Bewegungen sind um ein strategisches Framing bemüht (vgl. Benford/Snow 2000; Gamson 1992; Gerhards/ Rucht 1992; McAdam 1996). Framing beinhaltetet hier nicht nur die strategische Kommunikation der eigenen Sichtweise, bzw. des eigenen Frames, sondern auch den Wettbewerb mit den Frames anderer Kommunikatoren. In diesem Zusammenhang wird ein Frame definiert als „an idea through which political debate unfolds, and political alignment and collective action takes place“ (Pan/Kosicki 2001: 39).

(2) Beim journalistischen Framing werden Frames als „particular way in which journalists compose a news story to optimize audience assessibility“ aufgefasst (Valkenburg/Semetko/de Vreese 1999: 550). Beispielsweise zeigen Brosius/Eps (1993) sowie Scheufele (2003), wie journalistische Frames die Auswahl von Nachrichten beeinflussen. 
(3) Bei einem Großteil der Untersuchungen zum Framing-Ansatz steht die inhaltsanalytische Erfassung von Medien-Frames im Vordergrund. Ein Medien-Frame kann mit Gamson und Modigliani (1987: 143) zunächst allgemein als „central organizing idea or story line that provides meaning to an unfolding strip of events" umschrieben werden. Diese Untersuchungen beschäftigen sich ausschließlich mit der Deskription der Medienberichterstattung (vgl. Harden 2002; Miller/Andsager/Riechert 1998; Semetko/Valkenburg 2000; Simon/Xenos 2000).

(4) Im Vergleich zu den inhaltsanalytischen Studien gibt es zurzeit eher wenige Arbeiten, die die Wirkung von Medien-Frames auf Rezipienten-Frames untersuchen. Entman (1993: 53) begreift Rezipienten-Frames als „mentally stored clusters of ideas that guide individuals' processing of information“. Diese Forschungsbemühungen lassen sich wiederum in folgende Gruppen einteilen: das Second-Level Agenda Setting (vgl. McCombs 1992; McCombs 2000), experimentelle Wirkungsstudien (vgl. Iyengar 1991; Nelson/Kinder 1996) sowie qualitativ orientierte Wirkungsstudien (vgl. Gamson 1992; Gamson/Modigliani 1989; Neuman/Just/Crigler 1992).

Der folgende Beitrag beschäftigt sich nur mit einer der erwähnten Forschungsrichtungen: der inhaltsanalytischen Erfassung so genannter Medien-Frames. Der Fokus liegt dabei weniger auf einer theoretischen Diskussion des gesamten Frame-Ansatzes, vielmehr wird das Problem der reliablen und validen Erfassung von Medien-Frames aufgegriffen. Dieses Problem wird in zahlreichen Arbeiten zwar immer wieder konstatiert (vgl. Miller 1997: 376; Scheufele 1999: 103; Gandy 2001: 360-361; Hertog/McLeod 2001: 153; Tankard 2001: 104; Bonfadelli 2002: 152), allerdings nicht weiter erörtert. Auf einen Punkt gebracht könnte man mit Maher (2001: 84) formulieren: „[framing] has proved to be an elusive concept to measure".

Zunächst werden die verschiedenen Methoden zur Erfassung von Medien-Frames erläutert und kritisch diskutiert (2). Nach diesem Überblick über die Forschung wird eine eigene Methode zur Frame-Analyse vorgeschlagen, die sowohl eine höhere Inhaltsvalidität als auch eine höhere Reliabilität der Analyse verspricht und zudem besser als andere Verfahren in der Lage ist, die Veränderungen von Frames zu messen $(3,4)$. Im folgenden Abschnitt wird dann die empirische Umsetzung dieses Ansatzes an Hand einer international vergleichenden Studie zur Gentechnologieberichterstattung illustriert (5). Der Beitrag schließt mit einer kritischen Bewertung der vorgeschlagenen Operationalisierung von Medien-Frames und erörtert Schritte zu deren Verbesserung (6).

\section{Methoden zur Erfassung von Medien-Frames}

Zumeist werden in der Forschungsliteratur vereinfachend zwei methodische Zugänge zur Erfassung von Medien-Frames unterschieden: qualitative und quantitative FrameAnalysen (vgl. Hertog/McLeod 2001: 152, Miller 1997: 368; Reese 2001: 8; Scheufele 2003: 55 ff; Tankard 2001: 98). Die heuristische Einteilung in „qualitativ“ und "quantitativ“ ist wegen der Begriffsungenauigkeit für eine Beschreibung und Diskussion der Methoden zur Erfassung von Medien-Frames als unbefriedigend zu erachten (für die Vieldeutigkeit dieser Begriffe siehe u. a. Ponterotto/Grieger 1999: 50 f; Shadish 1995). Hier soll daher der Versuch unternommen werden, die Methoden an Hand ihrer charakteristischen Vorgehensweise einzuteilen. Insgesamt lassen sich so vier Methoden zur Erfassung von Medien-Frames unterscheiden:

1. textwissenschaftliche Verfahren,

2. interpretativ-quantifizierende Verfahren, 
3. computerbasierte quantitative Verfahren und

4. deduktive Verfahren.

In textwissenschaftlichen Untersuchungen werden die Frames nach der Auswahl, Platzierung und Strukturierung von Wörtern und Sätzen identifiziert (vgl. Entman 1991; Pan/Kosicki 1993). In der Regel erfolgt zunächst eine unstandardisierte Generierung von Frames auf der Basis weniger Texte. Diese Arbeiten gehen davon aus, dass „quantitative" Operationalisierungen die Bedeutung von Frames nicht vollständig erfassen könnten: „one must capture the meanings embedded in the internal relations within texts, which collapsing into reductive measures would obscure" (Reese 2001: 8). ${ }^{1}$ Den elaboriertesten textwissenschaftlichen Framing-Ansatz haben Pan und Kosicki (1993) vorgelegt. Von einem diskursanalytischen Modell ausgehend unterscheiden die Autoren vier Organisationsstrukturen von Nachrichtentexten: syntaktische, thematische und rhetorische Strukturen sowie Skripts. Syntaktische Strukturen beziehen sich auf makrosyntaktische Eigenschaften eines Textes, wie z. B. Überschrift, Vorspann, Schluss. Unter die thematische Struktur fallen Behauptungen oder Hypothesen zu einem Problem. Skripts sind Erzählstrukturen eines Beitrages. Schließlich sind mit den rhetorischen Strukturen stilistische Variationen des Autors gemeint. In diese Kategorie fallen auch die fünf von Gamson und Modigliani (1989) aufgeführten ,Framing-Devices': Metaphern, Beispiele, Schlüsselsätze, Schilderungen und Bilder. Resultat einer Frameanalyse ist eine Datenmatrix für jeden einzelnen Artikel, die für jede einzelne Proposition die verschiedenen „signifying elements“ (Pan/Kosicki 1993: 63) aufzeigt. Ein Vorteil dieser Herangehensweise ist sicherlich, dass Nachrichtentexte systematisch empirisch analysiert bzw. zergliedert werden können. Der inhaltsanalytische Aufwand für derartige Textanalysen ist allerdings entsprechend hoch anzusiedeln. Reese (vgl. 2001: 16) und Harden (vgl. 2002: 75) merken des Weiteren an, dass bei der textwissenschaftlichen Analyse nicht klar ist, wie und aus welchen Elementen sich ein Frame zusammensetzt. Tankard (2001: 98) schlussfolgert: „This approach makes frame identification a rather subjective process".

In einer anderen Gruppe von Untersuchungen werden die Frames in einem ersten Schritt vom Forscher selbst an einer Auswahl des Untersuchungsmaterials interpretativ generiert und anschließend qua Inhaltsanalyse kodiert und quantifiziert (vgl. AkhavanMajid/Ramaprasad 1998; Eilders/Lüter 2000; Hanson 1995; Harden 2002; Meyer 1995; Simon/Xenos 2000; Weßler 1999). Aufgrund dieses Vorgehens wird hierfür die Bezeichnung interpretativ-quantifizierendes Verfabren gewählt. Die Güte dieser Untersuchungen steht und fällt mit dem gewählten methodischen Vorgehen bei der induktiven Frame-Bildung sowie mit der Transparenz der Darstellung. Ein möglicher Nachteil ist die oftmals nicht explizierte und damit nicht nachvollziehbare Identifizierung der Frames (vgl. etwa Simon/Xenos 2000; Tucker 1998). Diese „Lücke“ spiegelt sich auch in den Beschreibungen wider, wie die Frames gewonnen wurden: „What emerged from the analysis of editorials was an anticolonial frame“ (Hanson 1995: 384) oder „I identify three master frames“ (Meyer 1995: 178) oder „The qualitative assessment of framing was based on careful reading" (Akhavan-Majid/Ramaprasad 1998: 144). Wenn - wie in den meisten Studien dieser Kategorie - nicht klar ist, wie und anhand welcher Kriterien die

1 Auch die Arbeiten von Gerhards (1993) sowie Gerhards und Rucht (1992) fallen unter diese Kategorie, obwohl es hier weniger um Medien-Frames als um das strategische Framing von sozialen Bewegungen geht. Das Framing wird bei Gerhards (vgl. 1993: 126 f) durch die Analyse der Argumentationsstruktur eines einzigen Textes analysiert. 
Frames identifiziert werden, fällt die Generierung der Frames in eine methodologische „Black Box“.2 Kurzum: Es besteht die Gefahr, dass „Forscher-Frames“ und nicht Medien-Frames erfasst werden. In der inhaltsanalytischen Literatur wird dieses Problem zwar mit Bezug auf die Kategorienbildung aufgegriffen, und es werden konstruktive Lösungsvorschläge unterbreitet (vgl. Bilandzic/Koschel/Scheufele 2001; Früh 1998: 134 ff); die Frame-Forschung aber ist auf diesem Auge bisher eher als blind zu bezeichnen. Ein weiterer Nachteil der interpretativ-quantifizierenden Methode ist, dass dieses Verfahren die Erfassung von zeitlich neuen oder kulturell andersartigen Frames erschwert (vgl. Kohring/Matthes 2002: 144). Wenn die Forscher oder Kodierer die kompletten Frames eigenhändig in einer qualitativen Vorstudie generieren, ist letztlich nicht auszuschließen, dass spezifische Erwartungen entwickelt werden, die die Einordnung und Kategorisierung von neuen Informationen steuern. Wirth (2001: 163) spricht in diesem Zusammenhang von Kodiererschemata. Dies führt dazu, dass in Längsschnittanalysen nur noch die relative Zunahme oder Abnahme bereits beobachteter Frames festgestellt und eine Analyse von Frame-Veränderungen sowie die Identifikation neuer Frames erheblich erschwert wird.

Auch Miller (Miller 1997: 376) weist auf die Notwendigkeit von objektiven und reliablen Methoden hin (vgl. auch Miller und Riechert 2001; Miller/Andsager/Riechert 1998). Hierfür schlagen Miller und Kollegen eine eigene quantitative Methode - das so genannte Frame-Mapping - vor. Bei diesem Verfahren werden Frames mit Hilfe von datenreduzierenden Verfahren aus dem gesamten Textmaterial extrahiert. Die Autoren greifen hierbei auf die Feststellung von Entman (1993) zurück, dass Frames sich anhand der Wortwahl eines Textes identifizieren lassen. Folglich verstehen Miller und Riechert (2001: 63) das Frame-Mapping als „efficient and precise method of probing for the particular words that occur together in some texts and do not tend to occur in other texts, then precisely quantifying and comparing those sets of terms that manifest distinctive frames“. Beim Frame-Mapping werden zusammen auftretende Wörter durch Computeralgorithmen identifiziert. Dieses Verfahren, so Miller (vgl. 1997: 368), mache die Frame-Analyse weitaus weniger subjektiv als die oben beschriebenen textwissenschaftlichen und interpretativ-quantifizierenden Arbeiten. In einer Frame-Mapping-Studie vergleichen Miller, Andsager und Riechert (vgl. 1998) Frames aus Pressemitteilungen über republikanische Primary-Präsidentschaftskandidaten mit den Frames aus Zeitungsbeiträgen (New York Times, Washington Post und Los Angeles Times) über diese Kandidaten. Insgesamt wurden in dieser Studie 28 (!) verschiedene Frames identifiziert. So setzten sich beispielsweise die Wörter charity, charities, charitable und money zu einem so genannten Charity-Frame zusammen. Hertog und McLeod (vgl. 2001: 152) geben zu bedenken, dass sehr wichtige Wörter in einem Text nicht sehr oft auftreten müssen, sie aber dennoch zentral für einen Frame seien können. Vom methodischen

2 Eine Ausnahme bilden in gewisser Hinsicht die Studie von Harden (2002) und Scheufele (2001; 2003). Harden (2002) generiert die Frames und deren Elemente qualitativ und beschreibt sie ausführlich. Anschließend werden die Elemente kodiert und faktorenanalytisch zusammengefasst. Die von Scheufele (2003) vorgeschlagene, aufwändige Frame-Identifizierungs-Technik (FIT) zur Erfassung journalistischer Kognitionen mag zwar reliabel sein, allerdings bleibt die Validität ungewiss. Es ist nämlich zu fragen, inwieweit man valide von einer Inhaltsanalyse verschiedener Artikel auf Schemata einzelner Journalisten schließen kann. Der Autor merkt selbst an, dass Journalistenbefragungen für zukünftige Untersuchungen in Betracht zu ziehen wären (vgl. ebd.: 218). 
Standpunkt aus entsteht allerdings der Eindruck, dass die Objektivität des Frame-Mappings auf Kosten der Inhaltsvalidität geht. Bei den extrahierten Wortclustern handelt es sich um sehr allgemeine Beschreibungen von Themenkomplexen, die anschließend als „Frames“ interpretiert werden (vgl. Gandy 2001: 361). Welche Wörter bzw. welche Elemente auftreten müssen, damit von einem Frame zu sprechen ist, wird nicht angegeben.

Eine Gemeinsamkeit aller bisher beschriebenen Anätze ist das induktive Vorgehen, d. h. die Frames werden erst nach Ansicht des empirischen Materials gewonnen. In deduktiven Untersuchungen wird der Versuch unternommen, Frames zunächst z. B. aus der Forschungsliteratur theoretisch abzuleiten und danach inhaltsanalytisch zu kodieren (vgl. Semetko/Valkenburg 2000; de Vreese/Peter/Semetko 2001). So extrahieren Semetko und Valkenburg (2000) aus der bisherigen Forschungsliteratur fünf Frames (Conflict, Human interest, Economic consequences, Morality und Responsibility). AnschlieBend wurden zu diesen Frames insgesamt 20 spezifische Fragen abgeleitet und für jeden untersuchten Medienbeitrag inhaltsanalytisch nominal kodiert. Beispielsweise wurde der Economy-Frame u. a. mittels der Frage „Is there a reference to economic consequences?" erfasst (vgl. Semetko/Valkenburg 2000: 100). Mit Hilfe von explorativen Faktoren- und Clusteranalysen konnten die postulierten Frames bestätigt werden. ${ }^{3}$ Eine Voraussetzung für das deduktive Vorgehen ist jedoch, dass die vorab ausgewählten Frames auch auf den jeweiligen Untersuchungsgegenstand bzw. auf das jeweilige Thema übertragen werden können. Semetko/Valkenburg (2000) unterscheiden daher zwischen themenspezifischen und generischen Frames. Generische Frames sind auf verschiedene Themen zu verschiedenen Zeiten und in verschiedenen kulturellen Kontexten anwendbar. Im Gegensatz dazu erlauben themenspezifische Frames eine detaillierte Analyse von besonderen Ereignissen. Eine deduktive Frame-Analyse ist aber nicht auf generische Frames beschränkt, auch themenspezifische Frames können vorab postuliert werden (vgl. Altheide 1997). Das oben angesprochene Problem der Objektivität und Reliabilität bei der Generierung von Frames besteht beim deduktiven Vorgehen nicht, allerdings können nur vorab bereits bekannte Frames untersucht werden.

Tabelle 1 gibt noch einmal einen Überblick über die bisherigen Methoden zur Erfassung von Medien-Frames mit ihren jeweiligen Vorteilen und Nachteilen. Vorteil der textwissenschaftlichen Methode ist sicherlich ihre Ausführlichkeit und Detailliertheit. Allerdings ist der damit verbundene Aufwand aus forschungsökonomischer Sicht eher nachteilig (vgl. Harden 2002: 76). Die Verknüpfung von qualitativem und quantitativem Vorgehen bei interpretativ-quantifizierenden Untersuchungen kann - wenn sie adäquat umgesetzt wird - sicherlich als Vorteil bezeichnet werden. Allerdings ist in den meisten Studien die Objektivität und die Reliabilität schwer zu beurteilen. Bei quantitativen Verfahren wie dem Frame-Mapping ist dies wiederum kein Problem. Nur geht hier die methodische Präzision auf Kosten der Validität der Frames (vgl. Gandy 2001: 361). Vorteil des deduktiven Vorgehens ist die erzielte Vergleichbarkeit der Ergebnisse. Dennoch können mit dieser Methode neuartige Frames nicht erfasst werden. Wie gezeigt wurde, weisen alle dargestellten Methoden sowohl Vorteile als auch Nachteile auf. Der weiteren Methodenentwicklung kommt daher eine besondere Bedeutung zu, denn die Konsequenzen dieses Mangels an methodischer Präzision sind nicht zu unterschätzen: „The

3 Allerdings machen Faktorenanalysen von inhaltsanalytischen Itembatterien nur dann Sinn, wenn die Kodierer die Frames nicht kennen. Falls die Kodierer aber darüber informiert sind, welche Fragen z. B. den Economy-Frame messen, könnten sich auf Grund dieses Vorwissens hohe Korrelationen zwischen den Items ergeben. 
problems of achieving and assessing the reliability of content analysis continue to limit our ability to understand the production, distribution, and reception of symbolic frames" (Gandy 2001: 360).

Tabelle 1: Methoden zur Erfassung von Medien-Frames

\begin{tabular}{|c|c|c|c|c|}
\hline & $\begin{array}{l}\text { Textwissen- } \\
\text { schaftlicher } \\
\text { Ansatz }\end{array}$ & $\begin{array}{l}\text { Interpretativ- } \\
\text { quantifizierender } \\
\text { Ansatz }\end{array}$ & $\begin{array}{l}\text { Frame- } \\
\text { Mapping }\end{array}$ & $\begin{array}{l}\text { Deduktive } \\
\text { Frame- } \\
\text { analyse }\end{array}$ \\
\hline $\begin{array}{l}\text { Analyse- } \\
\text { einheit }\end{array}$ & Proposition & Artikel & Artikel & Artikel \\
\hline Methode & $\begin{array}{l}\text { rhetorische } \\
\text { Textanalyse }\end{array}$ & $\begin{array}{l}\text { qualitative Ge- } \\
\text { nerierung, danach } \\
\text { Quantifizierung }\end{array}$ & $\begin{array}{l}\text { computergestützte } \\
\text { quantitative } \\
\text { Inhaltsanalyse }\end{array}$ & $\begin{array}{l}\text { Kodierung vorab } \\
\text { bestimmter Frames }\end{array}$ \\
\hline $\begin{array}{l}\text { Statistische } \\
\text { Auswertung }\end{array}$ & keine & deskriptiv & $\begin{array}{l}\text { Frame Mapping/ } \\
\text { Clusteranalyse von } \\
\text { Wörtern }\end{array}$ & deskriptiv \\
\hline Vorteil & Ausführlichkeit & $\begin{array}{l}\text { Verknüpfung von } \\
\text { qualitativen und } \\
\text { quantitativen } \\
\text { Verfahren }\end{array}$ & $\begin{array}{l}\text { große Textmengen, } \\
\text { sehr hohe Reliabilität } \\
\text { und Objektivität }\end{array}$ & $\begin{array}{l}\text { hohe Generalisierbar- } \\
\text { keit, hohe Reliabilität } \\
\text { und Objektivität }\end{array}$ \\
\hline Nachteil & $\begin{array}{l}\text { hoher Analyseauf- } \\
\text { wand, eingeschränkte } \\
\text { Validität }\end{array}$ & $\begin{array}{l}\text { Reliabilität und } \\
\text { Objektivität unklar; } \\
\text { Identifikation neu } \\
\text { auftretender Frames } \\
\text { erschwert }\end{array}$ & $\begin{array}{l}\text { eingeschränkte } \\
\text { Validität }\end{array}$ & $\begin{array}{l}\text { eingeschränkte Validi- } \\
\text { tät: keine Identifikation } \\
\text { vorab nicht definierter } \\
\text { Frames }\end{array}$ \\
\hline
\end{tabular}

\section{Frames als Cluster einzelner Frame-Elemente}

Im Folgenden wird eine Methode zur Erfassung von Medien-Frames vorgeschlagen, die versucht, sowohl die Inhaltsvalidität von Frames sicherzustellen als auch eine objektive Erfassung von Frames zu ermöglichen. Die Grundidee ist die Folgende: Wenn man ein Frame als bestimmtes, unverwechselbares Muster eines Textes versteht, das sich aus mehreren, von Journalisten selektiv ausgewählten und von Rezipienten beobachtbaren Elementen zusammensetzt, dann kann man dieses Muster auch empirisch bestimmen. Der Ausgangspunkt dieses Verfahrens ist also nicht, den kompletten Frame direkt zu kodieren, sondern seine einzelnen Elemente. In einem ersten Schritt müssen demnach die Elemente eines Frames aus einer allgemeinen Frame-Definition abgeleitet werden. In der Frame-Forschung lassen sich ganz unterschiedliche Auffassungen von Frames finden (vgl. Reese/Gandy/Grant 2001). Hierbei liegt die Schwierigkeit darin, dass verschiedene Autoren mit Frames auf unterschiedlichem Abstraktionsniveau arbeiten (vgl. Gamson 2001: S. X). Überhaupt mangelt es an Kriterien, wann von einem Frame zu sprechen ist. Auf eine Systematisierung und theoretische Diskussion aller zentralen Definitionen von Frames muss hier leider verzichtet werden. Da der Fokus dieses Beitrages auf methodischen Aspekten liegt, soll vielmehr nur ein wichtiger Systematisierungsaspekt der Definitionen betrachtet werden: die operationale Präzision. Es lassen sich zwei Arten von Definitionen unterscheiden: analytische und operationale. Bei analytischen Definitionen erfolgt eine Bedeutungsanalyse des Begriffes, ohne Hinweise zu geben, wie der Begriff empirisch zu erfassen ist (vgl. Bortz/Döring 1995: 61). Eine operationale Definition hingegen „standardisiert einen Begriff durch die Angabe der Ope- 
rationen, die zur Erfassung des durch den Begriff bezeichneten Sachverhaltes notwendig sind, oder durch die Angabe der messbaren Ereignisse, die das Vorliegen des Sachverhaltes anzeigen“ (Ebd.: 63). Zentrale und häufig zitierte Definitionen wie die von Gamson und Modigliani („central organizing idea“; 1987: 143), Gitlin („principles of selection, emphasis, and presentation composed of little tacit theories about what exists, what happens and what matters“; 1980: 6) oder Pan und Kosicki („,idea through which political debate unfolds“, 2001: 39) fallen für eine empirische Umsetzung zu unspezifisch aus. Analytische Definitionen können zwar aus theoretischer Sicht sehr fruchtbar sein. Das eingangs beschriebene Problem, dass das Frame-Konzept ein „elusive concept to measure“ (Maher 2001: 84) ist, vermögen sie aber nicht zu lösen. Da aus derart allgemeinen Beschreibungen die konkrete Operationalisierung nur schwer abgeleitet werden kann, wird das Frame-Verständnis letztlich einer stillschweigenden Übereinkunft zwischen Leser und Forscher überlassen (vgl. Entman 1993: 52). Operationale Definitionen wurden z. B. von Harden (2002) und Entman (1993) vorgeschlagen. Entmans FrameDefinition wird im Folgenden als Arbeitsdefinition zu Grunde gelegt, um die hier vorgeschlagene Methode beispielhaft zu illustrieren. Das Verfahren ließe sich aber auch mit anderen operationalen Definitionen umsetzen - schließlich herrscht in der Frame-Forschung alles andere als Einigkeit über die Definition von Frames. Die Entman-Definition hat den Vorteil, dass sie auf Grund ihrer weiten Verbreitung sozusagen das Bindeglied zwischen verschiedenen Forschungsrichtungen ist und demnach auch als Grundlage für die immer wieder eingeforderten Integrationsbemühungen dienen kann. Entman (1993: 52) definiert einen Frame folgendermaßen:

"To frame is to select some aspects of a perceived reality and make them more salient in a communicating context, in such a way as to promote a particular problem definition, causal interpretation, moral evaluation, and/or treatment recommendation for the item described."

Nach Entman setzt sich ein Frame also aus einer Problemdefinition, einer kausalen Attribution, einer moralischen Bewertung und/oder einer Handlungsanweisung zusammen. Beispielsweise wäre eine Problemdefinition beim Thema Drogen die Zunahme von Beschaffungskriminalität, die kausale Attribution beschuldigte die Drogenpolitik der Bundesregierung, eine mögliche moralische Evaluation würde negativ ausfallen und Handlungsforderungen richteten sich auf eine schärfere Gesetzgebung. Versteht man einen Frame als empirisch bestimmbares Textmuster, müsste er sich demnach durch eine ganz spezifische Konstellation der vier von Entman beschriebenen Frame-Elemente auszeichnen (wobei auch das Nichtvorkommen eines Elements bedeutsam ist). Mit anderen Worten: Wir nehmen an, dass sich die empirischen Ausprägungen der als Variablen operationalisierten und mittels einer Inhaltsanalyse erfassten Frame-Elemente in einer je charakteristischen Weise gruppieren und so zu verschiedenen Mustern formen können. Sofern ein solches Muster über mehrere Texte hinweg identifiziert werden kann, soll von einem Frame die Rede sein. Die Identifikation der Muster resp. Frames erfolgt nicht wie in den meisten bisherigen Ansätzen über eine direkte inhaltsanalytische Codierung des Frames, sondern über das statistische Verfahren der hierarchischen Clusteranalyse (vgl. Lorr 1994). ${ }^{4}$ Geclustert werden dabei die Ausprägungen der als Variablen begriffenen Frame-Elemente. Das Ziel dieses Verfahrens ist die Gruppierung von

4 Für die Bestimmung von Frames wurde dieses Verfahren wohl zum ersten Mal von Schöberl (vgl. 1995) angewendet, und zwar mit dem Ziel, auch neu entstandene Frames in der Risikoberichterstattung identifizieren zu können. 
Artikeln, wobei die Unterschiede innerhalb einer Gruppe möglichst klein und zwischen den Gruppen möglichst groß sein sollen. Im Vergleich zu partitionierenden Clusteranalysen liegt der Vorteil der hier verwendeten hierarchischen Clusteranalyse darin, dass dieses Verfahren durch das so genannte Ellenbogenkriterium die Bestimmung der Clusteranzahl ermöglicht (vgl. Backhaus et al. 1996: 307 f; Lathrop/Wiliams 1987: 953 ff). Die hierarchische Clusteranalyse gibt dem Forscher also zumindest einen Hinweis, wie viele Cluster bzw. Frames zu bestimmen sind.

Die vorgeschlagene Methode lässt sich, wie bereits erwähnt, nicht nur auf die Entman-Definition anwenden, sondern auf jede Definition, die die Elemente eines Frames bestimmt. Das Problem der validen Erfassung von Frames wird auf diesem Weg nicht vollständig gelöst, es verlagert sich vielmehr auf die inhaltsanalytische Kodierung der einzelnen Frame-Elemente. Die Kodierung einzelner Inhaltsvariablen und hier selbst die von Attributionen oder Handlungsempfehlungen ist aber weitaus unkomplizierter und besser zu standardisieren als die direkte Kodierung eines kompletten Frames (vgl. Kohring/Matthes 2002: 145-146). Je manifester die zu kodierenden Kategorien sind, umso höher ist die Reliabilität (Früh 1998: 95 ff). Auf diese Weise wird die Reliabilität einer inhaltsanalytischen Analyse von Frames deutlich verbessert; nicht zuletzt auch deshalb, weil der Kodierer gar nicht weiß, an welchem Frame er gerade „arbeitet“, wenn er in einem Artikel für sich stehende, einzelne Variablen wie z. B. Akteure oder Themen kodiert. Deshalb dürfte auch der Einfluss der oben angesprochenen Kodiererschemata (vgl. Wirth 2001: 163) geringer sein als bei der Kodierung des kompletten Frames. Die höhere Reliabilität bei der Kodierung minderkomplexer Variablen führt somit auch zu einer höheren Reliabilität bei der Bestimmung der Frames.

In einer ersten Anwendung (vgl. Kohring/Matthes 2002) konnte die vorgeschlagene Methode zur Erfassung von Frames bereits umgesetzt werden. Bei dem Thema Biotechnologie ließ sich der dynamische Verlauf von Medien-Frames über sieben Jahre hinweg illustrieren, wobei im Vergleich zweier Berichterstattungsperioden nicht nur Frames stabil blieben, neue Frames hinzukamen und ein Frame „ausstarb“, sondern auch Ausdifferenzierungen und Fokussierungen von Frames zu beobachten waren (vgl. Kohring/Matthes 2002: 151). Allerdings handelt es sich bei dieser Studie um eine Sekundäranalyse von Daten, die eine vollständige Umsetzung der von Entman vorgeschlagenen Frame-Elemente nicht ermöglichte. Ziel des vorliegenden Beitrages (der ebenfalls eine Sekundäranalyse darstellt) ist die erneute, dieses Mal vollständige Umsetzung der Entman-Definition an einem anderen, differenzierteren Datensatz. Hierbei werden aber nicht Frames im Zeitverlauf untersucht und verglichen, sondern der Fokus richtet sich diesmal auf eine international vergleichende Analyse von Frames in mehreren Ländern.

\section{Frames in der internationalen Berichterstattung über Gentechnologie}

Die hier verwendeten Daten stammen aus der Studie „Gentechnologie in den internationalen Medien“ (vgl. Kohring/Görke/Ruhrmann 1999). Es handelt sich hierbei um eine systematische qualitativ-quantifizierende Inhaltsanalyse der Berichterstattung von meinungsführenden journalistischen Organen über Gentechnologie in Deutschland, Frankreich, England und den USA. In den USA waren dies die Nachrichtenmagazine Time und Newsweek, in Großbritannien The Economist sowie The Sunday Times und in Frankreich Le Nouvel Observateur sowie L'Express. In Deutschland wurden Der Spiegel und Die Zeit analysiert. In einer Vollerhebung vom 1. Juli 1991 bis zum 30. Juni 1996 wurden insgesamt 1.180 Artikel inhaltsanalytisch ausgewertet. Das für die Studie 
entwickelte Kategoriensystem mit insgesamt 96 Variablen ermöglicht eine vollständige Umsetzung der von Entman definierten Frame-Elemente. Ein Reliabilitätstest mit 15 Artikeln des Spiegel aus den Jahren 1994, 1995 und 1996 ergab für die in dieser Studie verwendeten Variablen nach Dichotomisierung eine durchschnittliche Intercoderreliabilität (zwei Kodierer) von .72 (nach Scott; vgl. Merten 1995: 305 f). Für die nachfolgenden Auswertungen steht die deutsche Medienberichterstattung im Vordergrund die anderen Länder werden zum Vergleich und zur Validierung der Frame-Lösungen herangezogen.

Tabelle 2 zeigt die Frame-Elemente nach Entman, die inhaltsanalytischen Kategorien der Studie, mit deren Hilfe wir sie operationalisierten, sowie die Ausprägungen dieser Kategorien, die von uns verwendet wurden. Alle teilweise schon zusammengefassten Ausprägungen der ursprünglichen Kategorien kodierten wir zu dichotomen Variablen um (vgl. Backhaus et al. 1996: 265-272). Da auf diese Weise jede Ausprägung einer mehrkategorialen Variable Variablenstatus erhält und sich somit die Anzahl der Variablen stark erhöht, gingen nur diejenigen Ausprägungen in die Clusteranalyse ein, die eine Häufigkeit (gültige Prozente) von mehr als $5 \%$ (Themen) bzw. $10 \%$ (Nutzenbewertung, Schadensbewertung, verantwortlicher Akteur für Nutzen, verantwortlicher Akteur für Schaden) aufweisen.

Das Frame-Element „Problemdefinition“ umfasst zum einen die am häufigsten genannten Hauptthemen, wie z. B. Landwirtschaft, Regulierung oder Ethik, zum anderen die häufigsten Nutzens- und Schadensbewertungen, jeweils spezifisch für das Hauptthema eines Artikels. Das Frame-Element „kausale Interpretation“ operationalisierten wir mithilfe der für den Schaden bzw. Nutzen verantwortlich gemachten Akteure. Die

Tabelle 2: Operationalisierung der Frame-Elemente nach Entman (1993)

\begin{tabular}{|c|c|c|}
\hline Frame-Elemente & Kategorien & ausgewählte Ausprägungen \\
\hline \multirow[t]{2}{*}{ Problemdefinition } & Hauptthemen & $\begin{array}{l}\text { Landwirtschaft, Humananwendung, } \\
\text { medizinische Forschung, Grundlagen- } \\
\text { forschung, Regulierung, Wirtschaft, Ethik }\end{array}$ \\
\hline & $\begin{array}{l}\text { Nutzen- und Schadens- } \\
\text { bewertung des Haupt- } \\
\text { themas }\end{array}$ & $\begin{array}{l}\text { Nutzen für Wirtschaft, Medizin, } \\
\text { Wissenschaft } \\
\text { Schaden für Ökologie, Medizin, Kultur, } \\
\text { Moral, Wirtschaft und diffuser Schaden }\end{array}$ \\
\hline $\begin{array}{l}\text { Kausale } \\
\text { Interpretation }\end{array}$ & $\begin{array}{l}\text { verantwortlicher Akteur } \\
\text { für Nutzen bzw. Schaden }\end{array}$ & $\begin{array}{l}\text { verantwortlich für Nutzen: Wissenschaft, } \\
\text { diffuser Akteur }\end{array}$ \\
\hline & & $\begin{array}{l}\text { verantwortlich für Schaden: Wissenschaft, } \\
\text { Industrie, Regierung, diffuser Akteur }\end{array}$ \\
\hline
\end{tabular}

\begin{tabular}{lll}
\hline $\begin{array}{l}\text { Moralische } \\
\text { Bewertung }\end{array}$ & zukünftige Akzeptanz & positive vs. negative Akzeptanz \\
\hline $\begin{array}{l}\text { Handlungs- } \\
\text { empfehlung }\end{array}$ & $\begin{array}{l}\text { Aufforderung zur } \\
\text { Ausführung bzw. } \\
\end{array}$ & Ausführung bzw. Unterlassung \\
& $\begin{array}{l}\text { Unterlassung } \\
\text { positive bzw. negative }\end{array}$ & positive vs. negative Prognose \\
& Prognose & \\
\hline
\end{tabular}


relevanten Ausprägungen dieser Kategorien sind für Schäden Wissenschaft, Industrie, Regierung und diffuse Akteure, für Nutzen nur Wissenschaft und diffuse Akteure ${ }^{5}$. Das Frame-Element „Moralische Bewertung" drückt sich in der positiven oder negativen Akzeptanz von Biotechnologie aus. ${ }^{6}$ Für das Frame-Element „Handlungsempfehlung“ standen uns Variablen zur Verfügung, mit denen die Aufforderung zur Ausführung oder Unterlassung einer konkreten biotechnologischen Anwendung erfasst wurde. Diese Variablen wurden in die Ausprägungen Ausführung bzw. Unterlassung umkodiert. Zusätzlich bezogen wir negative und positive Prognosen ${ }^{7}$ mit ein, die ebenfalls unter dem Aspekt der Handlungsempfehlung gelesen werden können.

Vor diesem Hintergrund ergeben sich nunmehr drei Forschungsfragen.

1. Lassen sich mithilfe der vorgeschlagenen Methode und des zugrundeliegenden Framekonzepts von Entman Frames identifizieren und trennscharf voneinander abgrenzen?

2. Wenn ja, wie unterscheiden sich Die Zeit und Der Spiegel im Framing des Themas Biotechnologie?

3. Ist es möglich, die Berichterstattung mehrerer Länder anhand dieser Frame-Operationalisierung miteinander zu vergleichen; wenn ja, wie unterscheiden sich die Frames der untersuchten Länder?

\section{Ergebnisse}

Zunächst wird das Ergebnis der Frame-Analyse für Deutschland dargestellt. Im Anschluss wird die Analyse auf alle Länder erweitert. Für jedes Land wurde hierfür eine hierarchische Clusteranalyse mit dem Ward-Verfahren durchgeführt. Das Ward-Verfahren kann als ein sehr guter Fusionierungsalgorithmus angesehen werden (vgl. Breckenridge 2000: 281; Morey/Blashfield/Skinner 1983: 325; für nominale Variablen vgl. Hands/Everitt 1987: 242). Die Bestimmung der Clusteranzahl erfolgt über das „Ellenbogen-Kriterium“ (s. o.): Der „Ellenbogen“ zeigt an, an welcher Stelle des Algorithmus ein überproportionaler Zuwachs des Heterogenitätsmaßes (quadrierter euklidischer Abstand) vorliegt (vgl. Gore 2000: 316). An der Stelle, wo sich das Heterogenitätsmaß zwischen Clusterlösungen deutlich erhöht, ist die Bildung von neuen Clustern abzubrechen. Die Heterogenitätsmaße für die Analyse der deutschen Berichterstattung lauten: 1024 (8 Cluster), 1061 (7 Cluster), 1100 (6 Cluster), 1141 (5 Cluster), 1202 (4 Cluster), 1265 (3 Cluster). Bis zum fünften Cluster steigt der Wert immer um ca. 40, die Differenz vom Wert des fünften Clusters zum Wert des vierten Clusters beträgt dann aber 61, die nächste Differenz wieder 63. Folglich werden fünf Cluster identifiziert. ${ }^{8}$

5 Ein diffuser Akteur ist nicht konkret oder gar nicht benannt. Beispiele sind „wir“, „es“ oder „man“ oder aber Artikel, in denen zwar ein Nutzen oder ein Schaden benannt werden, der hierfür verantwortliche Akteur aber nicht klar ersichtlich wird.

6 Ursprünglich auf einer 7er-Skala erfasst, wurde Akzeptanz in die Ausprägungen positiv oder negativ umkodiert.

7 Die ursprünglich auf Einzelthemen bezogenen Prognosen wurden so rekodiert, dass sie entweder eine positive oder aber eine negative Prognose ausdrückten.

8 Für die Bestimmung der Cluster-Anzahl hat sich noch keine standardisierte, objektive Prozedur durchgesetzt (vgl. aber die Vorschläge von Schweizer 1992 und Krolak-Schwerdt/Eckes 1992 sowie die Zusammenstellung von Everitt 1992: 73 f). Von daher empfiehlt es sich immer, 
Tabelle 3 zeigt die Mittelwerte der Variablen für jedes Cluster bzw. jeden Frame in der deutschen Gentechnologieberichterstattung (Die Zeit und Der Spiegel) von 1991 bis 1996. Stichprobenartig ausgeführte $\chi^{2}$-Tests bestätigten die Trennschärfe der Cluster. $\mathrm{Da}$ alle Variablen dichotomisiert wurden, kann jede Variable einen Mittelwert von $\mathrm{O}$ bis 1 haben. ${ }^{9}$

Tabelle 3: Mittelwerte der dichotomen Variablen pro Cluster/Frame

\begin{tabular}{lccccc}
\hline Variablen & 1 & 2 & 3 & 4 & 5 \\
\hline Thema: Landwirtschaft & 0,08 & 0,02 & 0,38 & 0,29 & 0,06 \\
Thema: Humananwendung & 0,07 & 0,52 & 0,02 & 0,03 & 0,03 \\
Thema: Medizinische Forschung & 0,18 & 0,27 & 0,11 & 0,06 & 0,09 \\
Thema: Grundlagenforschung Genom & 0,57 & 0,02 & 0,13 & 0,05 & 0,27 \\
Thema: Regulierung & 0,00 & 0,04 & 0,16 & 0,35 & 0,03 \\
Thema: Ethik & 0,00 & 0,02 & 0,07 & 0,06 & 0,39 \\
Thema: Wirtschaft & 0,00 & 0,00 & 0,03 & 0,00 & 0,00 \\
Nutzensbewertung: Wirtschaft & 0,01 & 0,02 & 0,17 & 0,32 & 0,00 \\
Nutzensbewertung: Medizin & 0,00 & 0,83 & 0,05 & 0,06 & 0,12 \\
Nutzensbewertung: Wissenschaft & 0,96 & 0,03 & 0,06 & 0,10 & 0,79 \\
Schadensbewertung: Ökologie & 0,01 & 0,00 & 0,07 & 0,16 & 0,03 \\
Schadensbewertung: Medizin & 0,00 & 0,06 & 0,03 & 0,19 & 0,03 \\
Schadensbewertung: Kultur & 0,00 & 0,02 & 0,04 & 0,05 & 0,73 \\
Schadensbewertung: Ethik & 0,00 & 0,17 & 0,02 & 0,11 & 0,00 \\
Schadensbewertung: Diffus & 0,00 & 0,12 & 0,03 & 0,03 & 0,09 \\
Schadensbewertung: Wirtschaft & 0,01 & 0,02 & 0,03 & 0,16 & 0,00 \\
Verantwortlich f. Nutzen: diffuse Akteure & 0,00 & 0,06 & 0,10 & 0,11 & 0,03 \\
Verantwortlich f. Nutzen: Wissenschaft & 0,98 & 0,83 & 0,20 & 0,26 & 0,97 \\
Verantwortlich f. Schaden: diffuse Akteure & 0,00 & 0,03 & 0,08 & 0,10 & 0,03 \\
Verantwortlich f. Schaden: Industrie & 0,01 & 0,01 & 0,07 & 0,16 & 0,00 \\
Verantwortlich f. Schaden: Wissenschaft & 0,01 & 0,40 & 0,04 & 0,31 & 0,88 \\
Verantwortlich f. Schaden: Regierung & 0,02 & 0,00 & 0,01 & 0,21 & 0,00 \\
Akzeptanz: negativ & 0,01 & 0,17 & 0,06 & 0,65 & 0,61 \\
Akzeptanz: positiv & 0,08 & 0,26 & 0,19 & 0,18 & 0,30 \\
Forderung: weiterführen & 0,13 & 0,37 & 0,26 & 0,34 & 0,67 \\
Forderung: aufhören & 0,03 & 0,13 & 0,09 & 0,61 & 0,27 \\
Prognose: positiv & 0,40 & 0,64 & 0,49 & 0,16 & 0,52 \\
Prognose: negativ & 0,12 & 0,20 & 0,11 & 0,77 & 0,39 \\
\hline N (= 478) & 105 & 126 & 152 & 62 & 33 \\
\% (=100) & 22,0 & 26,4 & 31,8 & 13,0 & 6,9 \\
\hline & & & & & \\
& & & &
\end{tabular}

sowohl die Clusteranzahl als auch den Fusionierungsalgorithmus zu variieren. Folglich haben wir alternative Lösungen für vier bzw. sechs Cluster berechnet. Bei der Lösung mit vier Clustern sind zwei Cluster der ursprünglichen Lösung „zusammengefallen“ und dadurch schwerer zu interpretieren. In der 6-Cluster Lösung „spaltete“ sich ein Cluster in zwei schwer interpretierbare Einzel-Cluster „auf“. Auch aus inhaltlicher Sicht war demnach die 5-Clusterlösung zu präferieren.

9 Mittelwerte von dichotomen Variablen sind mit Interpretationsschwierigkeiten verbunden, allerdings erleichtern sie hier den Überblick. 
Bei der Interpretation der Mittelwerte sind zwei Fragen zu berücksichtigen: Zum Ersten, welche Variablen innerhalb eines Clusters die höchsten Mittelwerte haben, und zum Zweiten, in welchen Clustern eine Variable ihre höchsten Mittelwerte hat. Beide Perspektiven sind wichtig, da auch ein geringer Wert von Bedeutung sein kann, wenn er relativ zu allen anderen Clustern hoch ist; und ein hoher Wert von geringerer Bedeutung, wenn er überall hoch ist (vgl. auch Hair et al. 1998: 508 ff).

Das erste Cluster, bzw. der erste Frame, wird hier als „Forschung in der Biomedizin“ $(22 \%, n=105)$ bezeichnet. In diesen Artikeln wird vor allem die Grundlagenforschung zum menschlichen Genom thematisiert, wobei auf den Nutzen der Forschung hingewiesen wird. Für diesen werden die Wissenschaftler verantwortlich gemacht. Mögliche Risiken werden in diesen Beiträgen nicht erwähnt. Des Weiteren werden weder Aussagen zur Akzeptanz der Biotechnologie geäußert, noch werden Forderungen aufgestellt. Der Biotechnologie wird eine positive Zukunft prognostiziert. Ein Viertel $(26 \%$, n = 126) der gesamten Berichterstattung beschäftigt sich mit dem medizinischen Aspekt der Gentechnologie. Dieser Frame wird „medizinischer Nutzen“ genannt. In diesen Artikeln wird den Anwendungen der Gentechnik am Menschen ein hoher medizinischer Nutzen zugesprochen: Dieser sei vor allem den Wissenschaftlern zuzuschreiben, wenngleich diese auch Risiken erzeugen würden. Am Rande werden moralische und eher diffuse Risiken mitangesprochen. Insgesamt wird diese Forschungsrichtung aber akzeptiert, und es wird deutlich zu ihrer Fortführung angehalten. Die Prognosen für die zukünftige Entwicklung fallen positiv aus. Der umfangreichste Frame wird als „Landwirtschaftlicher Nutzen“ bezeichnet (32\%, n = 152). Das Thema Biotechnologie wird hier in Hinsicht auf die Landwirtschaft diskutiert, wobei vor allem ein wirtschaftlicher Nutzen erwähnt wird. Mögliche Risiken werden nicht thematisiert. Auch die politische Regulierung der Biotechnologie wird angesprochen; gleichzeitig wird aber gefordert, diesen Forschungs- und Anwendungsbereich weiter zu verfolgen. Dementsprechend fallen auch die Prognosen für landwirtschaftliche Anwendungen der Gentechnologie positiv aus. Der vierte Frame „Landwirtschaft: Pro \& Contra“ $(13 \%, \mathrm{n}=62)$ betont die wirtschaftlichen Vorteile landwirtschaftlicher Anwendungen der Gentechnik - in keinem anderen Frame werden diese stärker hervorgehoben. Zugleich wird aber auf ökologische, medizinische, moralische und auch wirtschaftliche Risiken hingewiesen. Für die Risiken verantwortlich gemacht wird die Wissenschaft, dieser Vorwurf trifft aber auch Industrie und Regierung. Gefordert wird, landwirtschaftliche Anwendungen der Gentechnik zu unterlassen und eine Regulierung unter Beteiligung der Öffentlichkeit anzustreben. Befürchtet wird zudem, dass Regulierung und ethische Diskussion zu keinem Ergebnis führen. Aus struktureller Sicht handelt sich bei diesem Frame um einen typischen Konflikt-Frame, in dem gegensätzliche Akzeptanzurteile und Handlungsempfehlungen aufeinander treffen, wobei allerdings die ablehnenden überwiegen. In einem fünften Frame (7 \%; n = 33), den wir „Ethische Abwägung“ genannt haben, geht es um die ethische Reflexion der Gentechnologie, die sich aus dem Widerspruch von einerseits hohem wissenschaftlichen Nutzen und andererseits hohem kulturellen Risiko der Forschung speist. Die Wissenschaft sieht sich in der paradoxen Situation, zugleich die Nutzen als auch die Risiken angeblich allein zu verantworten. All dies drückt sich in einer fehlenden Akzeptanz der Gentechnologie und der Forderung nach einer ethischen Diskussion aus, nicht zuletzt wegen der Angst vor Biologismus und Eugenik. Auffallend ist, dass sich dieser relativ kleine Ethik-Frame auch in einer 4- oder 6-Cluster-Lösung als sehr robust erweist, d. h. es werden immer wieder die gleichen 33 Artikel identifiziert.

Tabelle 4 zeigt die Verteilung der Frames auf die beiden analysierten journalistischen Organe Der Spiegel und Die Zeit. Die Frames „Medizinischer Nutzen“, „Landwirt- 
Tabelle 4: Verteilung der Frames pro journalistisches Organ

\begin{tabular}{lrccc}
\hline Frames & \multicolumn{3}{c}{ Organ } \\
& & Der Spiegel & Die Zeit & Gesamt \\
\hline \multirow{2}{*}{ Forschung i. d. Biomedizin } & $\mathrm{n}$ & 31 & 74 & 105 \\
& $\%$ & 15,1 & 27,1 & 22,0 \\
Medizinischer Nutzen & $\mathrm{n}$ & 55 & 71 & 126 \\
& $\%$ & 26,8 & 26,0 & 26,4 \\
Landwirtschaftlicher Nutzen & $\mathrm{n}$ & 83 & 69 & 152 \\
& $\%$ & 40,5 & 25,3 & 31,8 \\
Landwirtschaft: Pro \& Contra & $\mathrm{n}$ & 21 & 41 & 62 \\
\multirow{4}{*}{ Ethische Abwägung } & $\%$ & 10,2 & 15,0 & 13,0 \\
& $\mathrm{n}$ & 15 & 18 & 33 \\
& $\%$ & 7,3 & 6,6 & 6,9 \\
& $\mathrm{n}$ & 205 & 273 & 478 \\
& $\%$ & 100 & 100 & 100 \\
\hline
\end{tabular}

schaft: Pro \& Contra“ sowie „Ethische Abwägung“ treten bei beiden Zeitungen ungefähr gleich häufig auf. Hingegen weist die Berichterstattung des Spiegel den Frame „Landwirtschaftlicher Nutzen“ mit 45,3 \% deutlich häufiger auf als Die Zeit (25,3 \%). Der Frame „Forschung in der Biomedizin“ tritt mit 27,1 \% dagegen häufiger in der Zeit auf als im Spiegel.

In einem zweiten Schritt sollen nun die für jedes Land getrennt ermittelten Clusterlösungen miteinander verglichen werden. In Tabelle 5 wird die deutsche Frame-Struktur quasi „gesetzt“, um von hier aus Gemeinsamkeiten und Unterschiede in der internationalen Gentechnologieberichterstattung in den Blick zu bekommen. ${ }^{10}$ Mittelwerttabellen werden aus Platzgründen nicht berichtet. Die Bestimmung der Clusteranzahl erfolgte ebenfalls über das Ellenbogen-Kriterium. Für die USA lassen sich 5 Cluster ${ }^{11}$, für Großbritannien 4 Cluster ${ }^{12}$ und für Frankreich ebenfalls 4 Cluster ${ }^{13}$ bestimmen. Als Erstes fällt hierbei die hohe Vergleichbarkeit der Berichterstattung anhand der ermittelten Textmuster auf. Der Frame „Forschung in der Biomedizin“ findet sich in der Berichterstattung aller untersuchten Länder, ebenso der Frame „Medizinischer Nutzen“. In den USA und in Großbritannien differenziert sich dieser Frame in zwei MedizinFrames aus, von denen einer mehr allgemein die medizinische Forschung thematisiert, während der andere sich vor allem (Großbritannien) bzw. auch (USA) mit Humananwendungen beschäftigt und hierbei auch Risiken anspricht. Es ist zu beachten, dass wir es in diesen Ländern mit unterschiedlichen Entwicklungsstadien der Gentechnologie zu tun haben. Während Anfang der neunziger Jahre in Deutschland unter anderem

10 Vgl. zu international vergleichenden Studien zur (deutschen) Gentechnologieberichterstattung auch Görke/Kohring/Ruhrmann 2000; Kohring/Görke 2000; Gutteling et al. 2002.

11 Heterogenitätsmaße USA: 357 (8 Cluster), 375 (7 Cluster), 394 (6 Cluster), 413 (5 Cluster), 448 (4 Cluster).

12 Heterogenitätsmaße Großbritannien: 402 (8 Cluster), 426 (7 Cluster), 451 (6 Cluster), 479 (5 Cluster), 510 (4 Cluster), 557 (3 Cluster).

13 Heterogenitätsmaße Frankreich: 389 (8 Cluster), 411 (7 Cluster), 435 (6 Cluster), 460 (5 Cluster), 487 (4 Cluster), 522 (3 Cluster). 
die strikte Gentechnikgesetzgebung von 1990 erörtert wurde und man wissenschaftspolitisch um Anschluss an die internationale Entwicklung bemüht war (vgl. Hampel/ Ruhrmann/Kohring/Görke 1998), wurden in Großbritannien neben wirtschaftlichen Aspekten auch schon ganz konkrete Risiken gentechnologischer Diagnosemöglichkeiten für „belastete“ Versicherungsnehmer diskutiert. In einem der beiden britischen „Medizinischer Nutzen“-Frames wird diese Thematik als kulturelles Risiko der Gentechnologie mitthematisiert. Der Frame „Landwirtschaftlicher Nutzen“ findet sich in Deutschland, in Großbritannien und in Frankreich. Die britische Berichterstattung weist als Einzige kein Ethik-Frame auf. Nur in Deutschland wiederum findet sich der Frame „Landwirtschaft: Pro \& Contra“. Im Gegensatz zu den Frames in der deutschen, britischen und französischen Berichterstattung ist die Lösung für die USA etwas unbefriedigend: In den USA besteht mit $23 \%$ rund ein Viertel der Berichterstattung aus einem Cluster, der nur durch einen relativ hohen Anteil positiver Prognosen für die Gentechnologie auffällt und ansonsten „von allem etwas“ enthält: In diesem Cluster werden sowohl die Themen Landwirtschaft und Medizinische Forschung als auch Grundlagenforschung und Regulierung behandelt. Eine kausale Attribution auf soziale Akteure erfolgt nicht. Diesen Frame haben wir wegen dieser (Nicht-)Eigenschaften „Diffuser allgemeiner Fortschritt" betitelt. Allerdings ist für die Interpretation eines Frames nicht nur entscheidend, welchen Ausschnitt der Realität der Frame fokussiert, sondern auch welchen er nicht fokussiert, also im Sinne des Wortes außen vor lässt. Der Frame „Diffuser allgemeiner Fortschritt" in der amerikanischen Berichterstattung thematisiert weder den Nutzen noch die Risiken der Gentechnologie. Folglich werden auch keine verantwortlichen Akteure für einen Nutzen oder Schaden ausgemacht. Im Prinzip werden in diesen Artikeln einzelne Themen der Biotechnologie vorgestellt und generell positiv

Tabelle 5: Frames in der internationalen Gentechnologieberichterstattung 1991-1996

\begin{tabular}{|c|c|c|c|c|}
\hline \multirow[t]{2}{*}{ Frames } & \multicolumn{4}{|c|}{ Länder } \\
\hline & $\mathrm{D}$ & UK & F & USA \\
\hline $\begin{array}{l}\text { Landwirtschaftlicher } \\
\text { Nutzen }\end{array}$ & $\begin{array}{c}32 \% \\
(\mathrm{n}=152)\end{array}$ & $\begin{array}{c}35 \% \\
(n=84)\end{array}$ & $\begin{array}{c}56 \% \\
(n=140)\end{array}$ & - \\
\hline $\begin{array}{l}\text { Medizinischer } \\
\text { Nutzen }\end{array}$ & $\begin{array}{c}26 \% \\
(n=126)\end{array}$ & 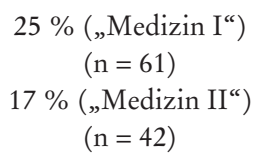 & $\begin{array}{c}21 \% \\
(n=53)\end{array}$ & $\begin{aligned} & 25 \%(\text { („Medizin I“) } \\
&(\mathrm{n}=52) \\
& 17 \%(\text { („Medizin II“) } \\
&(\mathrm{n}=36)\end{aligned}$ \\
\hline $\begin{array}{l}\text { Forschung in der } \\
\text { Biomedizin }\end{array}$ & $\begin{array}{c}22 \% \\
(n=105)\end{array}$ & $\begin{array}{c}22 \% \\
(n=54)\end{array}$ & $\begin{array}{c}16 \% \\
(n=41)\end{array}$ & $\begin{array}{c}22 \% \\
(n=47)\end{array}$ \\
\hline $\begin{array}{l}\text { Landwirtschaft: } \\
\text { Pro \& Contra }\end{array}$ & $\begin{array}{c}13 \% \\
(n=62)\end{array}$ & $\begin{array}{l}- \\
-\end{array}$ & $\begin{array}{l}- \\
-\end{array}$ & $\begin{array}{l}- \\
-\end{array}$ \\
\hline Ethische Abwägung & $\begin{array}{c}7 \% \\
(n=33)\end{array}$ & - & $\begin{array}{c}7 \% \\
(n=17)\end{array}$ & $\begin{array}{c}12 \% \\
(n=26)\end{array}$ \\
\hline $\begin{array}{l}\text { Diffuser allgemeiner } \\
\text { Fortschritt }\end{array}$ & - & - & - & $\begin{array}{c}23 \% \\
(n=49)\end{array}$ \\
\hline & $\begin{array}{c}100 \\
(478)\end{array}$ & $\begin{array}{c}100 \\
(241)\end{array}$ & $\begin{array}{c}100 \\
(251)\end{array}$ & $\begin{array}{c}100 \\
(210)\end{array}$ \\
\hline
\end{tabular}


bewertet, wobei eine Nutzen-Schaden-Diskussion vollkommen vermieden wird. Obgleich es möglich ist, dass in der Berichterstattung solche Muster erscheinen, könnte es sich hier auch um ein Problem einer Sekundäranalyse handeln, in der man es mit nicht spezifisch für das Untersuchungsziel operationalisierten Variablen zu tun hat. Dennoch ist es insgesamt als ein positives Ergebnis zu werten, dass sich mit dem Verfahren der Clusteranalyse in vier verschiedenen Samples gleiche Frames identifizieren lassen. Dies kann als ein Beleg für die in der Forschungsliteratur häufig geforderte Validierung einer einmal (hier für die deutsche Berichterstattung) vorgenommenen Cluster- bzw. FrameBestimmung gedeutet werden (vgl. Breckenridge 2000: 261; Gore 2000: 317; Hair et al. 501; Lorr 1994: 180; Morey/Blashfield/Skinner 1983: 327; Punj/Stewart 1983: 12; Speece 1994: 41).

\section{Diskussion}

Für die bisherige Frame-Forschung lassen sich nicht nur eine konzeptionelle Vielfalt und eine daraus resultierende begriffliche Unklarheit konstatieren, sondern auch methodische Probleme bei der empirischen Erfassung von Frames. In diesem Beitrag wurden vier Verfahren der Frame-Analyse vorgestellt und deren spezifische Vorteile und Nachteile diskutiert. Textanalytische Studien beschäftigen sich mit Textstrukturen, anhand derer ein Frame identifiziert werden kann. Allerdings erfolgt die Analyse nur auf der Grundlage weniger Texte, was die Verallgemeinerbarkeit und damit Brauchbarkeit für die kommunikationswissenschaftliche Forschung erheblich einschränkt (vgl. Harden 2002: 76). Bei interpretativ-quantifizierenden Studien werden die Frames an einem Ausschnitt des Untersuchungsmaterials generiert und anschließend quantifiziert. Nachteil dieses Herangehens kann eine mangelnde Objektivität sein, wenn die Generierung der Frames nicht offen gelegt wird und damit nicht nachvollziehbar ist. Rein quantitative Verfahren bestimmen die Frames mit einer computerbasierten Inhaltsanalyse, die gemeinsam auftretende Wörter identifiziert. Das Problem bei diesen Verfahren ist die Interpretation dieser Wörtersammlungen als Frames. Schließlich werden bei deduktiven Studien Frames a priori bestimmt und inhaltsanalytisch kodiert. Dieses Verfahren ist allerdings nur für die Analyse bereits bekannter Frames brauchbar.

Der vorliegende Beitrag schlägt eine Methode zur Erfassung von Medien-Frames vor, die sowohl eine objektive als auch eine detaillierte Frame-Analyse ermöglicht (wobei die detaillierte Analyse hier auf Deutschland beschränkt wurde). Demonstriert wurde das Verfahren beispielhaft an der gängigen Frame-Definition von Entman, die die Elemente eines Frames eindeutig bestimmt und hier als Arbeitsdefinition verwendet wurde. Die Methode ist aber nicht auf das damit zugrunde gelegte Frame-Verständnis beschränkt. Die hier vorgeschlagene Methode geht davon aus, dass sich die durch eine Inhaltsanalyse erfassten Frame-Elemente in einer charakteristischen Weise gruppieren und so zu einem Muster formen. Wenn dieses Muster über mehrere Texte hinweg identifiziert werden kann, dann kann man von einem Frame sprechen. Der Hauptunterschied zu bisherigen Methoden liegt darin, dass Frames nicht direkt inhaltsanalytisch kodiert, sondern die einzelnen Ausprägungen der Frame-Elemente erst clusteranalytisch zu Frames zusammengefasst werden. Der entscheidende Vorteil dieser Methode ist eine stärkere Forscherunabhängigkeit und damit eine höhere Reliabilität der Ergebnisse, da sich einzelne Elemente reliabler kodieren lassen als der gesamte Frame. Zudem können in Längsschnittstudien zeitlich oder kulturell andersartige bzw. neue Frames leichter identifiziert werden (vgl. Kohring/Matthes 2002). Nicht zuletzt ist diese Methode vergleichsweise einfach zu handhaben. Schließlich sind wegen der in vielen In- 
haltsanalysen zumeist standardmäßigen Kodierung von Frame-Elementen vielfach sekundäranalytische Frame-Bestimmungen möglich (wie hier geschehen). Die vorgeschlagene Methode konnte in dieser Studie erneut umgesetzt werden (vgl. für die vorherige Umsetzung Schöberl 1995; Kohring/Matthes 2002). Es konnten für die deutsche, englische, französische sowie teilweise auch für die US-amerikanische Gentechnologieberichterstattung verschiedene Frames identifiziert werden. Allerdings zeigte sich in der US-amerikanischen Berichterstattung ein Cluster von Artikeln, die sich nur schwer als Frame interpretieren ließen. Dies könnte zum einen auf den sekundäranalytischen Charakter der Untersuchung zurückzuführen sein. Zum anderen wirft dieses Ergebnis aber die Frage auf, ob jeder untersuchte Beitrag überhaupt einen Frame im Sinne Entmans aufweisen muss. Denkbar wäre nämlich auch, dass es Cluster von Artikeln gibt, die eher als Non-Frames zu bezeichnen wären (vgl. Harden 2002: 190).

Allerdings können sich bei dem hier vorgestellten Verfahren auch Anwendungsprobleme ergeben: Beispielsweise kann das in dieser Studie verwendete nominale Datenniveau aufgrund des hohen Informationsverlustes und der damit verbundenen Interpretationsschwierigkeiten nicht als optimal bezeichnet werden. Eine gute Alternative zu diesem Vorgehen ist das z-Standardisieren von Variablen mit unterschiedlichem Skalenniveau (vgl. Schaffer/Green 1996). Entgegen dem Vorgehen von Harden (2002) und Semetko/Valkenburg (2000) ist aber mit Cromrey (vgl. 1988: 758) davon abzuraten, nominalskalierte Variablen mit einer explorativen Faktorenanalyse zu reduzieren (vgl. allerdings McLeod/Swygert/Thissen 2001). Zudem gilt zu bedenken, dass das Ward-Verfahren eher zu gleich großen Clustern neigt (vgl. Lorr 1994: 185). Zwar ließen sich auch in einem kleinen Sample (z. B. 30 Artikel) clusteranalytisch Frames identifizieren, allerdings ist es eher unwahrscheinlich, dass zugleich sehr „kleine“ und sehr „große“ Frames extrahiert werden. Außerdem ist die Selektion der Variablen entscheidend für die Clusterlösung. Eine sekundäranalytische Auswahl wie in dieser Studie ist daher sicherlich mit Nachteilen verbunden. Auch wenn zu viele Variablen ausgewählt werden, wird die Identifizierung der Cluster schwieriger (vgl. Speece 1994: 37 f). Des Weiteren ist nicht davon auszugehen, dass das Ellenbogenkriterium - als gewissermaßen heuristische Entscheidung - in jeder Analyse eine Interpretation der Clusteranzahl ermöglicht (vgl. Krolak-Schwerdt/ Eckes 1992: 542). In diesem Fall empfiehlt sich ein sukzessives Testen von verschiedenen Clusterlösungen mit unterschiedlichen Algorithmen (vgl. Hair et al. 1998: 499). Mit Oldenbürger (vgl. 1981: 406) ist aber davor zu warnen, dass die Clusteranalyse „nach persönlicher Vorliebe und in einer schwer nachvollziehbaren Auswahl ihrer Resultate nach einem Kriterium der ,theoretischen Angemessenheit' bzw. ,Interpretierbarkeit' erfolgt." Schließlich muss der Anwender viele kritische methodische Entscheidungen treffen, für die es keine objektiven Richtlinien gibt (vgl. Gore 2001: 298; Everitt 1992: 141; Oldenbürger 1981: 425 f; Punj/Stewart 1983: 11). Es können jegliche, auch zufällige Daten und Variablen analysiert und mit einer Clusteranalyse ausgewertet werden: „The investigator must be concerned whether the resulting clusters are discovered or forced by the technique" (Speece 1994: 36). Eine empirische Frame-Analyse, die sich dieses Verfahrens bedient, sollte daher die Auswahl der Frame-Elemente sowie deren Operationalisierung explizit begründen und im methodischen Teil die gewählte Clusterlösung erörtern (vgl. Punj/Stewart 1983: 10). Für ein exploratives Verfahren wie die Clusteranalyse erscheint es überdies unabdingbar, dass die Clusterlösung an einem unabhängigen Sample repliziert und validiert wird (vgl. Breckenridge 2000: 282). Für die Erfassung von Medien-Frames bietet es sich an, die Frame-Analyse für verschiedene Subgruppen des gleichen Samples durchzuführen, um die Konsistenz der Ergebnisse zu überprüfen (vgl. Everitt 1992: 143). 
Auch eine ganze Reihe weiterer Kernprobleme des Frame-Konzeptes müssen hier unbeantwortet bleiben: Beispielsweise haben die Medien-Frames in der Forschungsliteratur einen unterschiedlichen Abstraktionsgrad. Prinzipiell lassen sich strukturelle und inhaltliche (themenspezifische und generische) Frames unterscheiden (vgl. McCombs/Ghanem 2001: 71; Semetko/Valkenburg 2000). In diesem Beitrag wurden allerdings nur themenspezifische Frames untersucht. Bei strukturellen Frames steht hingegen die Präsentationsform einer Medienbotschaft im Vordergrund, d. h. es geht darum, wie eine Nachricht aufgebaut ist. Die von Iyengar (1991) unterschiedenen thematischen und episodischen Frames fallen in diese Kategorie. Überdies herrscht in der Frame-Forschung noch keine Einigkeit darüber, ob jedem Text ein einziger oder mehrere Frames zugeordnet werden sollen. Nicht zuletzt gilt es auch, den FramingAnsatz aus seinem Status eines ,ill-defined concept“ (Pan/Kosicki 2001: 38, Fußnote 4) herauszulösen, um somit auch die für die Cluster-Analyse zu operationalisierenden Frame-Elemente unmissverständlich definieren zu können (s. o.). Hierfür sollten die eingangs erwähnten Forschungszweige stärker verknüpft werden. Nur so wird sich auch zeigen, inwieweit der Frame-Ansatz sein ihm zugeschriebenes integratives Potenzial auszuschöpfen vermag. Aus Sicht der Medienwirkungsforschung steht hier vor allem noch die empirische Verknüpfung von Medien-Frames mit „Rezipienten-Frames“ aus.

\section{Literatur}

Akhavan-Majid, Roya/Ramaprasad, Jyotika (1998): Framing and ideology: A comparative analysis of U.S. and Chinese newspaper coverage of the Fourth United Nations Conference on Women and the NGO Forum. In: Mass Communication \& Society, 1. Jg., 3/4, S. 131-152.

Altheide, David L (1997): The news media, the problem frame, and the production of fear. In: Sociological Quarterly, 38. Jg., S. 647-668.

Backhaus, Klaus/Erichson, Bernd/Plinke, Wulff/Weiber, Rolf (1996): Multivariate Analysemethoden. Eine anwendungsorientierte Einführung. Achte, verbesserte Auflage. Berlin/Heidelberg/New York.

Benford, Robert D./Snow, David A. (2000): Framing processes and social movements: An overview and assessment. In: Annual Review of Sociology, 26. Jg., S. 611-639.

Bilandzic, Helena/Koschel, Frederike/Scheufele, Bertram (2001): Theoretisch-heuristische Segmentierung im Prozeß der empiriegeleiteten Kategorienbildung. In: Wirth, Werner/Lauf, Edmund (Hrsg.): Inhaltsanalyse: Perspektiven, Probleme, Potentiale. Köln, S. 98-116.

Bonfadelli, Heinz (2002): Medieninhaltsforschung. Konstanz.

Bortz, Jürgen/Döring, Nicola (1995): Forschungsmethoden und Evaluation. Berlin.

Breckenridge, James N. (2000): Validating cluster analysis: Consistent replication and symmetry. In: Multivariate Behavioral Research, 35. Jg., S. 261-285.

Brosius, Hans Bernd/Eps, Peter (1993): Verändern Schlüsselereignisse journalistische Selektionskriterien? Framing am Beispiel der Berichterstattung über Anschläge gegen Ausländer und Asylanten. In: Rundfunk und Fernsehen, 41. Jg., S. 512-530.

Cromrey, Andrew L. (1988): Factor-analytic methods of scale development in Personality and Clinical Psychology. In: Journal of Consulting and Clinical Psychology, 56. Jg., S. 754-761.

Eilders, Christiane/Lüter, Albrecht (2000): Germany at war: competing framing strategies in German public discourse. In: European Journal of Communication, 15. Jg., S. 415-428.

Entman, Robert M. (1993): Framing: toward clarification of a fractured paradigm. In: Journal of Communication, 43. Jg., Nr. 4, S. 51-58.

Everitt, Brian S. (1992): Cluster analysis. New York.

Früh, Werner (1998): Inhaltsanalyse. Theorie und Praxis. 4., überarbeitete Auflage. München.

Gamson, William A. (1992): Talking politics. Cambridge. 
Gamson, William A. (2001): Foreword. In: Reese, Stephen D./Gandy, Oscar H./Grant, August E. (Hrsg.): Framing public life: perspectives of media and our understanding of the social world. Mahwah (NJ), S. 139-161.

Gamson, William A./Modigliani, Andre (1987): The changing culture of affirmative action. In Braungart, Richard G./Braungart, Margaret M. (Hrsg.): Research in political sociology. Greenwich (CT), S. 137-177.

Gamson, William A./Modigliani, Andre (1989): Media discourse and public opinion on nuclear power: a constructionist approach. In: American Journal of Sociology, 95. Jg., S. 1-37.

Gandy, Oscar H. Jr. (2001): Epilogue-Framing at the horizon: a retrospective assessment. In: Reese, Stephen D./Gandy, Oscar H./Grant, August E. (Hrsg.): Framing public life: perspectives of media and our understanding of the social world. Mahwah (NJ), S. 355-378.

Gerhards, Jürgen (1993): Neue Konfliktlinien in der Mobilisierung öffentlicher Meinung: eine Fallstudie. Opladen.

Gerhards, Jürgen/Rucht, Dieter (1992): Mesomobilization: organizing and framing in two protest campaigns in West Germany. In: American Journal of Sociology, 98. Jg., S. 555-595.

Gitlin, Todd (1980): The whole world is watching: mass media in the making and unmaking of the new left. Berkeley.

Gore, Paul A. Jr. (2000): Cluster analysis. In: Tinsley, Howard E. A./Brown, Steven D. (Hrsg.): Handbook of applied multivariate statistics and mathematical modeling. San Diego, S. 298-321.

Görke, Alexander/Kohring, Matthias/Ruhrmann, Georg (2000): Gentechnologie in der Presse. Eine internationale Langzeitanalyse von 1973 bis 1996. In: Publizistik, 45. Jg., S. 20-37.

Gutteling, Jan M./Olofsson, Anna/Fjæestad, Björn/Kohring, Matthias/Görke, Alexander/Bauer, Martin W./Rusanen, Timo (2002): Media coverage 1973 - 1996: trends and dynamics. In: Bauer, Martin W./Gaskell, George (Hrsg.): Biotechnology - the making of a global controversy. Cambridge, S. 95-128.

Hair, Joseph F. Jr./Anderson, Rolph E./Tatham, Ronald, L./Black, William C. (1998): Multivariate data analysis. New Jersey.

Hampel, Jürgen/Ruhrmann, Georg/Kohring, Matthias/Görke, Alexander (1998): Germany. In: Durant, John/Bauer, Martin W./Gaskell, George (Hrsg.): Biotechnology in the public sphere. A European sourcebook. London, S. 63-76.

Hands, Stephen/Everitt, Brian (1987): A Monte Carlo study of the recovery of cluster structure in binary data by hierarchical clustering techniques. In: Multivariate Behavioral Research, 22. Jg., S. 235-243.

Hanson, Elizabeth C. (1995): Framing the world news: The Times of India in changing times. In: Political Communication, 12. Jg., S. 371-393.

Harden, Lars (2002): Rahmen der Orientierung. Eine Längsschnittanalyse von Frames in der Philosophieberichterstattung deutscher Qualitätsmedien. Opladen.

Hertog, James K./McLeod, Douglas M. (2001): A multiperspectival approach to framing analysis: a field guide. In: Reese, Stephen D./Gandy, Oscar H./Grant, August E. (Hrsg.): Framing public life: perspectives of media and our understanding of the social world. Mahwah (NJ), S. 139-161.

Iyengar, Shanto (1991): Is anyone responsible? How television frames political issues. Chicago.

Kohring, Matthias/Görke, Alexander (2000): Genetic engineering in the international media: an analysis of opinion-leading magazines. In: New Genetics and Society, 19. Jg., S. 345-363.

Kohring, Matthias/Görke, Alexander/Ruhrmann, Georg (1999): Das Bild der Gentechnologie in den internationalen Medien - eine Inhaltsanalyse meinungsführender Zeitschriften. In: Hampel, Jürgen/Renn, Ortwin (Hrsg.): Gentechnik in der Öffentlichkeit. Wahrnehmung und Bewertung einer umstrittenen Technologie. Frankfurt am Main/New York, S. 313-339.

Kohring, Matthias/Matthes, Jörg (2002): The face(t)s of biotech in the nineties: how the German press framed modern biotechnology. In: Public Understanding of Science, 11. Jg., S. $143-154$.

Krolak-Schwerdt, Sabine/Eckes, Thomas (1992): A graph theoretic criterion for determining the number of clusters in a data set. In: Multivariate Behavioral Research, 27. Jg., S. 541-565. 
Lathrop, Richard G./Wiliams, Janice E. (1987): The reliability of inverse scree tests for cluster analysis. In: Educational \& Psychological Measurement, 47. Jg., S. 953-959.

Lorr, Maurice (1994): Cluster analysis: aims, methods, problems. In: Strack, Stephen/Lorr, Maurice (Hrsg.): Differentiating normal and abnormal psychology. New York, S. 179-195.

Maher, Michael T. (2001): Framing: an emerging paradigm or a phase of agenda setting? In: Reese, Stephen D./Gandy, Oscar H./Grant, August E. (Hrsg.): Framing public life: perspectives of media and our understanding of the social world. Mahwah (NJ), S. 83-94.

McAdam, Doug (1996): The framing function of movement tactics: strategic dramaturgy in the American civil rights movement. In: McAdam, Doug/McCarthy, John D./Zald, Mayer N. (Hrsg.): Comparative perspectives on social movements: political opportunities, mobilizing structures, and cultural framings. New York, S. 338-355.

McCombs, Maxwell (2000): Agenda Setting: Zusammenhänge zwischen Massenmedien und Weltbild. In: Schorr, Angela (Hrsg.): Publikums- und Wirkungsforschung. Opladen, S. $123-136$.

McCombs, Maxwell E. (1992): Explorers and surveyors: Expanding strategies for agenda-setting research. In: Journalism Quarterly, 69. Jg., S. 813-824.

McCombs, Maxwell/Ghanem, Salma I. (2001): The convergence of agenda setting and framing. In: Reese, Stephen D./Gandy, Oscar H./Grant, August E. (Hrsg.): Framing public life: perspectives of media and our understanding of the social world. Mahwah (NJ), S. 67-81.

McCombs, Maxwell/Llamas, Juan Pablo/Lopez-Escobar, Esteban/Rey, Federico (1997): Candidate images in Spanish elections: second-level agenda-setting effects. In: Journalism \& Mass Communication Quarterly, 74. Jg., S. 703-717.

McLeod, Lori D./Swygert, Kimberly A./Thissen, Davis (2001): Factor analysis for items scored in two categories. In: Thissen, David/Wainer, Howard (Hrsg.): Test scoring. Mahwah (NJ), S. $189-216$.

Merten, Klaus (1995): Inhaltsanalyse. Einführung in Theorie, Methode und Praxis. 2., verbesserte Auflage. Opladen.

Meyer, David S. (1995): Framing national security: elite public discourse on nuclear weapons during the Cold War. In: Political Communication, 12. Jg., S. 173-192.

Miller, Mark M. (1997): Frame mapping and analysis of news coverage of contentious issues. In: Social Sience Computer Review, 15. Jg., S. 367-378.

Miller, Mark/Andsager, Julie/Riechert, Bonnie P. (1998): Framing the candidates in presidential primaries. In: Journalism \& Mass Communication Quarterly, 75. Jg., S. 312-324.

Miller, Mark/Riechert, Bonnie P. (2001): Frame mapping: a quantitative method for investigating issues in the public sphere. In: West, Mark D. (Hrsg.): Theory, method, and practice in computer content analysis. Norwood (NJ), S. 61-76.

Morey, Leslie C./Blashfield, Roger K./Skinner, Harvey A. (1983): A comparison of cluster analysis techniques within a sequential validation framework. In: Multivariate Behavioral Research, 18. Jg., S. 309-329.

Nelson, Thomas E./Kinder, Donald R. (1996): Issue framing and group-centrism in American public opinion. In: Journal of Politics, 58. Jg., S. 1055-1078.

Neuman, W. Russell/Just, Marion R./Crigler, Ann N. (1992): Common knowledge: news and the construction of political meaning. Chicago.

Oldenbürger, Hartmut A. (1981): Clusteranalyse. In: Bredenkamp, Jürgen/Feger, Hubert (Hrsg.): Enzyklopädie der Psychologie (Themenbereich B, Serie I, Bd. 4). Göttingen, S. 391439.

Pan, Zhongdang/Kosicki, Gerald M. (1993): Framing analysis: an approach to news discourse. In: Political Communication, 10. Jg., S. 55-76.

Pan, Zhongdang/Kosicki, Gerald M. (2001): Framing as a strategic action in public deliberation. In: Reese, Stephen D./Gandy, Oscar H./Grant, August E. (Hrsg.): Framing public life: perspectives of media and our understanding of the social world. Mahwah (NJ), S. 35-65.

Ponterotto, Joseph G./Grieger, Ingrid (1999): Merging qualitative and quantitative perspectives in a research identity. In: Kokala, Mary/Suzuki, Lisa A. (Hrsg.): Using qualitative methods in psychology. Thousand Oaks, S. 49-62. 
Punj, Girish/Stewart, Dawid W. (1983): Cluster analysis in marketing research: review and suggestions for application. In: Journal of Marketing Research, 20. Jg., S. 134-148.

Reese, Stephen D. (2001): Prologue - Framing public life: a bridging model for media research. In: Reese, Stephen D./Gandy, Oscar H./Grant, August E. (Hrsg.): Framing public life: perspectives of media and our understanding of the social world. Mahwah (NJ), S. 7-31.

Reese, Stephen D./Gandy, Oscar H./Grant, August E. (Hrsg.) (2001): Framing public life: perspectives of media and our understanding of the social world. Mahwah (NJ).

Schaffer, Catherine M./Green, Paul E. (1996): An empirical comparison of variable standardization methods in cluster analysis. In: Multivariate Behavioral Research, 31. Jg., S. 149-167.

Scheufele, Bertram (2001): Notwendigkeit, Nutzen und Aufwand von Mehrfach- und Sonderkodierungen. In: Wirth, Werner/Lauf, Edmund (Hrsg.): Inhaltsanalyse: Perspektiven, Probleme, Potentiale. Köln, S. 82-97.

Scheufele, Bertram (2003): Frames - Framing - Framing-Effekte. Theoretische und methodische Grundlegung des Framing-Ansatzes sowie empirische Befunde zur Nachrichtenproduktion. Opladen.

Scheufele, Dietram A. (1999): Framing as a theory of media effects. In: Journal of Communication, 49. Jg., S. 103-122.

Schöberl, Markus (1995): Frames in der Risikoberichterstattung. Unveröffentlichte Magisterarbeit. Westfälische Wilhelms-Universität Münster, Institut für Publizistik- und Kommunikationswissenschaft (Prof. Weischenberg).

Schweizer, Karl (1992): A correlation based decision-rule for determining the number of clusters and its efficiency in uni- and multi-level data. In: Multivariate Behavioral Research, 27. Jg., S. 77-94.

Semetko, Holli A./Valkenburg, Patti M. (2000): Framing European politics: a content analysis of press and television news. In: Journal of Communication, 50. Jg., S. 93-109.

Shadish, William R. (1995): Philosophy of science and the quantitative-qualitative debates: thirteen common errors. In: Evaluation and Program Planning, 18. Jg., S. 63-75.

Simon, Adam/Xenos, Michael (2000): Media framing and effective public deliberation. In: Political Communication, 17. Jg., S. 613-624.

Speece, Deborah, L. (1994): Cluster analysis in perspective. In: Exceptionality, 5. Jg., S. 31-44.

Tankard, James W. (2001): An empirical approach to the study of media framing. In: Reese, Stephen D./Gandy, Oscar H./Grant, August E. (Hrsg.): Framing public life: perspectives of media and our understanding of the social world. Mahwah (NJ), S. 95-106.

Tucker, Lauren R. (1998): The framing of Calvin Klein. In: Critical Studies in Mass Communication, 15. Jg., S. 141-157.

Valkenburg, Patti M./Semetko, Holli A./Vreese, Claes H. de (1999): The effects of news frames on readers' thoughts and recall. In: Communication Research, 26. Jg., S. 550-569.

Vreese, Claes H. de/Peter, Jochen/Semetko, Holli A. (2001): Framing politics at the launch of the Euro: a cross-national comparative study of frames in the news. In: Political Communication, 18. Jg., S. 107-122.

Weßler, Hartmut (1999): Öffentlichkeit als Prozeß. Deutungsstrukturen und Deutungswandel in der deutschen Drogenberichterstattung. Opladen.

Wirth, Werner (2001): Der Codierprozeß als gelenkte Rezeption. Bausteine für eine Theorie des Codierens. In: Wirth, Werner/Lauf, Edmund (Hrsg.): Inhaltsanalyse: Perspektiven, Probleme, Potentiale. Köln, S. 157-182. 